\title{
Isomeria: Uma descoberta de Jacob Berzelius sob o olhar de Larry Laudan
}

\author{
Isomeria: A discovery by Jacob Berzelius under the eye of Larry Laudan \\ Isomería: Un descubrimiento de Jacob Berzelius bajo la mirada de Larry Laudan
}

\section{Resumo}

Neste trabalho buscou-se aproximar a epistemologia de Larry Laudan, que traz como pressuposto que o progresso científico está baseado na Resolução de Problemas, ao assunto de Isomeria, trabalho de pesquisa realizado por Jacob Berzelius. Este tema tem relevância no estudo da Química Orgânica, pois a isomeria é um fenômeno comum e retrata a diversidade de moléculas orgânicas que apresentam a mesma fórmula molecular, mas com propriedades e características estruturais diferentes. No entanto, seu ensino em sala de aula vem ocorrendo de forma fragmentada devido à dificuldade encontrada por professores e alunos em desenvolver a abstração necessária para compreender as suas mais diversas representações. Objetiva-se, a partir da compreensão da importância e da efetividade da descoberta realizada por Berzelius, relacionada à epistemologia de Larry Laudan, proporcionar que o assunto Isomeria possa ser abordado em sala de aula de forma mais compreensível, procurando, desta forma, minimizar as dificuldades entre o ensino e a aprendizagem.

Palavras-chave: Isomeria; Jacob Berzelius; Larry Laudan.

\begin{abstract}
In this work, we sought to bring Larry Laudan's epistemology, which presupposes that scientific progress is based on Problem Solving, closer to the subject of Isomerism, a research carried out by Jacob Berzelius. This theme is relevant in the study of Organic Chemistry, as isomerism is a common phenomenon and portrays the diversity of organic molecules that have the same molecular formula, but different structural properties and characteristics. However, its teaching in the classroom has been occurring in a fragmented way due to the difficulty found by teachers and students in developing the necessary abstraction to understand its most diverse representations. This work aims from the understanding of the importance and effectiveness of the discovery made by Berzelius, related to Larry Laudan's epistemology, to provide that the subject Isomerism can be approached in the classroom in a more understandable way, seeking, in this way, to minimize the difficulties between teaching and learning.
\end{abstract}

Keywords: Isomerism; Jacob Berzelius; Larry Laudan.

\section{Resumen}

En este trabajo se buscó acercar la epistemología de Larry Laudan, que presupone que el progreso científico se basa en la Resolución de Problemas, al tema de Isomeria, un trabajo de investigación realizado por Jacob Berzelius. Este tema es relevante en el estudio de la Química Orgánica, ya que la isomería es un fenómeno común y retrata la diversidad de moléculas orgánicas que tienen la misma fórmula molecular, pero tienen diferentes propiedades y características estructurales. Sin embargo, su enseñanza en el aula se viene dando de forma fragmentada debido a la dificultad que encuentran docentes y alumnos para desarrollar la abstracción necesaria para comprender sus más diversas representaciones. El objetivo es desde la comprensión de la importancia y efectividad del descubrimiento hecho por Berzelius, relacionado con la epistemología de Larry Laudan, para proporcionar que el tema Isomeria pueda ser abordado en el aula de una manera más comprensible, buscando, de esta manera, minimizar las dificultades entre la enseñanza y el aprendizaje.

Palabras clave: Isomeria; Jacob Berzelius; Larry Laudan. 


\section{Introdução}

Isômeros são dois ou mais compostos diferentes que apresentam a mesma fórmula molecular (Atkins \& Jones, 2012). A origem dessa definição iniciou-se em 1830, quando Jacob Berzelius realizava uma síntese orgânica e observou a existência de compostos que apresentavam a mesma fórmula molecular, porém com propriedades físicas e químicas distintas (Correa et al., 2010). Da reunião do prefixo grego iso equivalendo a mesmo e meros equivalendo a partes iguais, nomeou-se os compostos encontrados como isômeros, dando sentido ao que os químicos Justus von Liebig e Friedrich Wöhler já haviam identificado alguns anos antes.

Correa et al. (2010) comentam que este fenômeno é menos frequente nos compostos inorgânicos, sendo assim, considerada uma qualidade própria das substâncias orgânicas. Observa-se que suas propriedades químicas não dependem unicamente de sua composição, mas também do arranjo espacial dos átomos dentro da molécula, que são essenciais para a determinação das propriedades físico-químicas das mesmas e favorecidas pelo grande número de combinações possíveis entre os átomos de carbono.

Este assunto encontra relevância no estudo da Química Orgânica, pois a isomeria é um fenômeno comum e retrata a diversidade de compostos presentes na natureza e na vida cotidiana, como por exemplo, nos fármacos, nos quais a utilização do isômero adequado é útil para inúmeros tratamentos farmacológicos. No passado, usavam-se misturas de isômeros e, hoje, sabe-se que o isômero inadequado pode causar sérios danos à saúde, existindo fármacos de isômeros conhecidos usados ainda na farmacoterapia e outros que tiveram seu uso proibido devido aos riscos causados à saúde, como o medicamento utilizado nos anos 60 chamado Talidomida que em sua estrutura trazia um carbono quiral e, assim, apresentava dois isômeros ópticos que agiam simultaneamente e causou uma grande tragédia pelo seu uso (Leite; Silva \& Lins, 2019).

Infelizmente, ainda nos dias atuais, seu ensino em sala de aula vem ocorrendo de forma fragmentada devido às dificuldades encontradas por professores e alunos em desenvolver a abstração necessária para compreender as suas mais diversas representações (Marcelino et al., 2010). É notório que os processos de ensino e aprendizagem em escolas brasileiras, particulares como públicas, sofrem uma série de dificuldades ocasionadas pela carência de recursos sejam pedagógicos e/ou tecnológicos que favoreçam aos alunos uma boa compreensão das estruturas moleculares e, assim, dificultam o aprendizado de assuntos que se relacionam a esta temática como o conteúdo de isomeria (Vieira et al., 2019). Conforme as Orientações Curriculares para o Ensino Médio (Brasil, 2006), estimular o uso da História da Ciência, como uma das maneiras de minimizar as dificuldades de aprendizagem em diversas áreas da Química, pode ser um caminho possível para compreender o conceito científico.

Para entender a importância e a efetividade da descoberta realizada por Berzelius, pode-se lançar mão da epistemologia de Larry Laudan (1986) que afirma que o progresso científico efetivamente ocorre quando os problemas não resolvidos e/ou anômalos são transformados em problemas resolvidos, devendo questionar-se quantos problemas determinada teoria resolveu e quais anomalias ela enfrenta. Esta questão torna-se uma ferramenta de fundamental importância para a avaliação comparativa entre teorias científicas.

Na sociedade percebe-se que existem muitos produtos que são resultados do crescimento científico e tecnológico. Muitas vezes esses avanços não são percebidos, mas estudiosos e pensadores contemporâneos trazem à tona esta percepção, através da reflexão sobre o papel da Ciência e da tecnologia na Resolução de Problemas e que resultam em impacto positivo à humanidade (Vilela et al., 2021).

Desta forma, neste trabalho busca-se aproximar a epistemologia de Larry Laudan, a qual indica que o progresso científico baseia-se na Resolução de Problemas, ao trabalho de pesquisa realizada por Jacob Berzelius que nominou o assunto isomeria. Desta forma, acredita-se encontrar uma estratégia útil para minimizar as dificuldades que esta matéria encontra ao ser abordada em sala de aula e assim facilitar o ensino e a aprendizagem. 


\section{Metodologia}

Neste trabalho, foi realizada uma pesquisa de natureza qualitativa, trazendo uma discussão teórica sobre as reflexões entre um fato histórico, um conteúdo de Química e a metodologia de Resolução de Problemas como evolução do conhecimento científico e facilitador no ensino aprendizagem. A historicidade das evoluções científicas encontra relação com a epistemologia da Ciência no sentido de melhor interpretar o papel de ser formador na área de Ciências da Natureza (Vilela et al., 2021).

A pesquisa de natureza qualitativa costuma procurar no ambiente natural suas fontes de dados e tem o pesquisador como principal instrumento, sugerindo que, para um melhor resultado, ocorra um contato direto e prolongado com o objeto pesquisado (Ludke \& André, 1986).

Neste sentido, Santana e Lemos (2018) trazem ao debate de pesquisa em educação que a abordagem qualitativa supera seus principais obstáculos e procura alternativas para serem utilizadas no processo de busca do conhecimento, com olhar para a resolução de situações práticas do contexto educacional.

No intuito de desenvolver este trabalho, procurou-se realizar um estudo bibliográfico que consiste na investigação em material teórico sobre o assunto de interesse que, conforme GIL (2007), tem como principais exemplos as investigações sobre ideologias ou aquelas que se propõem à análise das diversas posições acerca de um problema. Este material pode ser encontrado em vários formatos, como livros, revistas, vídeos, ou seja, todo tipo de fonte que possa remeter ao objeto de estudo investigado (Cervo \& Bervian, 2002).

\section{Tradição Investigativa de Larry Laudan}

Nascido em 1945 nos Estados Unidos, formou-se Bacharel em Física na Universidade de Kansas e realizou mestrado e doutorado em Filosofia na Universidade de Princeton, Ministrou aulas de História e Filosofia das Ciências em várias universidades. Dentre suas obras, destacam-se Progress and Its Problems (1977); Science and Hypothesis (1981); Science and Values (1984); Science and Relativism (1990) e Beyond Positivism and Relativism (1996).

Na obra Progress and Its Problems (1977), Laudan apresenta sua epistemologia, evidenciando o resgate da racionalidade da Ciência, buscando evitar alguns dos pressupostos-chaves que produziram o que ele chama de "colapso da análise tradicional' (Ostermann et al., 2008). Neste livro, Laudan apresenta que as tradições de investigação são as metodologias, as técnicas que perduram através da mudança de teorias e estabelecem o que existe de continuidade na História da Ciência. Laudan defende que a coexistência de tradições de investigação rivais e de teorias rivais é uma regra para o avanço da Ciência, em clara oposição a Kuhn o qual defende a existência de um único paradigma na fase de ciência normal, cuja fronteira é a revolução científica (Moreira \& Massoni, 2011).

Moreira e Massoni (2011) defendem que, sob a perspectiva de Laudan, os estudos sobre o desenvolvimento histórico da Ciência têm mostrado que ela tem sido um empreendimento racional. Porém, encontra-se associada a alguns traços persistentes como: não ser acumulativo; não se refutar teorias por suas anomalias; suas mudanças e controvérsias poderem ser resolvidas conceitualmente; o princípio da racionalidade apresentar mudança com o tempo; e a regra passar a ser a existência de teorias rivais, tal que a evolução das teorias possa apresentar-se como uma atividade comparativa.

Em sua trajetória, Larry Laudan teve a oportunidade de ser aluno ou colega de vários nomes que contribuíram para formar o caráter da História e da Filosofia da Ciência contemporânea como: Hempel, Kuhn, Buchdal, Feyrabend, Popper, Lakatos e Grünbaum e isto lhe proporcionou, poder ser crítico a algumas ideias destes mesmos estudiosos (Laudan, 1986). Suas ideias evoluem a partir das críticas às obras de Thomas Kuhn (1978) e Imre Lakatos (1989). Segundo Laudan (1986), o primeiro representa o abandono da racionalidade da Ciência e o segundo, uma pequena variação na análise tradicional.

Assim, segundo Laudan, uma tradição de pesquisa é um conjunto de teorias em evolução que não podem ser analisadas fora de seu contexto histórico e possuem duas características, a primeira é a metodológica, que diz respeito ao grupo 
de regras do que é permitido fazer e o que não é em cada área, e a segunda é a ontológica, que se refere aos objetos de estudo da tradição (Laudan, 1986).

Laudan (1986) afirma que há três funções importantes da tradição de pesquisa: a de determinar os limites de aplicação das teorias que constituem uma área; a de proporcionar ideias iniciais que permitam a formulação de teorias explicativas, preditivas e verificáveis e a de justificar de maneira racional a existência de teorias científicas. A avaliação da tradição de pesquisa é feita por meio de comparação entre a tradição vigente e a nova. A tradição que promover o maior número de problemas resolvidos será considerada progressiva, sendo que deste modo, será avaliada apenas a capacidade que a tradição tem em resolver problemas, ou seja, os quesitos verdade e falsidade de seus pressupostos metafísicos e metodológicos não serão levados em conta.

$\mathrm{Na}$ impossibilidade de julgamento entre uma teoria condizente com a verdade e outra concorrente, não é possível dizer que a Ciência tem sido progressiva nestes termos, mas pode se dizer que a Ciência se encaminha a produzir teorias bem comprovadas, que têm aplicação prática e que conseguem predizer fatos novos. Laudan (1986) propõe que o objetivo da Ciência é produzir teorias eficazes na resolução de problemas, ou um modelo científico por resolução de problemas, concluindo que, se os problemas são o foco do pensamento científico, as teorias são o seu resultado final, havendo dois tipos de problemas: os empíricos e os conceituais.

Problema empírico é qualquer coisa do mundo natural que nos surpreenda como estranha e que necessite de uma explicação. Define-se problemas empíricos como aqueles de primeira ordem, cujas soluções pressupõem estudos dos objetos de um determinado estado de coisas real, ou, pelo menos, pensados como estado de coisa real. Ou seja, não há problema empírico livre de teorias, sendo estes mais fáceis de exemplificar do que de definir (Laudan, 1986).

Para Laudan (1986), há três tipos de problemas empíricos: a) problemas não resolvidos, que nenhuma teoria resolveu adequadamente e servem de estímulos para novas investigações; b) problemas resolvidos adequadamente por uma teoria, ou seja, que confirmam a mesma; e c) problemas anômalos, que são resolvidos por uma ou mais teorias alternativas e são constituintes de provas contra certa teoria. É importante, em todos os casos, observar as teorias comparadas e questionadas sob o panorama de quantos problemas resolveu e quantas anomalias surgiram.

A solução de problemas não deve ser confundida com a explicação de fatos. Conforme Laudan (1986), um problema empírico está resolvido quando um determinado contexto de investigação não mais é considerado uma pergunta sem resposta e uma teoria resolve o problema em questão. Já nos problemas não resolvidos, a sabedoria convencional julga estimular o crescimento e o progresso da Ciência estabelecendo credenciais científicas assim que se tornam resolvidos. Os problemas anômalos, por sua vez, resumem-se em problemas reais que as teorias rivais resolvem, mas não foram resolvidos pela teoria em questão (Laudan, 1986).

O problema conceitual é um problema apresentado por alguma teoria, das quais respondem perguntas de ordem superior acerca da estrutura e consistência conceitual das teorias, sendo que estas foram criadas para responder perguntas de primeira ordem.

Os problemas conceituais podem apresentar-se de dois tipos, os internos e os externos. O problema conceitual interno mostra-se quando a teoria apresenta inconsistências internas, ambiguidades ou bases de análise pouco claras os quais confrontam princípios da tradição de investigação à qual pertence. O problema conceitual externo aparece quando a teoria contradiz pressupostos metafísicos, teorias, doutrinas ou metodologias dominantes, colocando-se em conflito com essas metodologias (Laudan, 1986).

Santos e Goi (2012) relatam que Laudan reconhece que os problemas conceituais são gerados por três tipos de dificuldades, chamadas Intra-Científicas, normativas e relativas à visão de mundo. Nas Dificuldades Intra-Científicas existem tensões entre teorias, de modo que, na existência de duas teorias inconsistentes, pelo menos uma delas será abandonada. Nas 
Dificuldades Normativas existem tensões entre a metodologia vigente e as teorias científicas, trazendo nas evidências históricas, teorias com forte relação com a metodologia vigente e nesse sentido constituindo problemas conceituais que, uma vez solucionados, geram modificações nas teorias ou nas metodologias. Por último, Dificuldades relativas à Visão de Mundo apresentam crenças científicas versus crenças não científicas, buscando incompatibilidades das teorias com crenças amplamente aceitas e que vão além dos domínios da ciência.

Laudan (1986) entende que a História da Ciência mostra que muitos dos grandes debates entre os cientistas defensores de teorias rivais têm ocorrido no campo conceitual, ainda que os problemas conceituais não tenham encontrado lugar nos principais modelos epistemológicos, sendo este um dos meios importantes para o desenvolvimento da Ciência.

Os problemas conceituais podem, ainda, ser de ordem interna ou externa. Os de ordem interna ocorrem quando uma teoria é logicamente inconsistente e, portanto, autocontraditória, associados a ambiguidades ou circularidades, que normalmente exigem um melhor esclarecimento da teoria. Já os problemas conceituais de ordem externa mostram-se quando uma teoria é logicamente inconsistente com outra teoria aceita.

O progresso científico efetivamente ocorre quando os problemas não resolvidos e/ou anômalos são transformados em problemas resolvidos. Devem-se perguntar então quantos problemas determinada teoria resolveu e quais anomalias ela enfrenta. Esta questão torna-se uma ferramenta de fundamental importância para a avaliação comparativa entre teorias científicas (Laudan, 1986).

Neste sentido, Medeiros et al., (2020) refletem que a Epistemologia defendida por Larry Laudan apresenta-se como um referencial teórico apropriado para ser trabalhado no Ensino de Ciência, pois um enfoque filosófico e histórico da Ciência mostra-se relevante ao trabalho científico. A epistemologia de Laudan pode contribuir no contexto escolar para o entendimento de teorias específicas de modo que esses entendimentos permitam a alunos e professores a superação do senso comum e, assim, ultrapassar barreiras para a compreensão das Ciências.

\section{Tradição Investigativa de Jacob Berzelius}

Nascido em Estocolmo, no ano de 1779, Jöns Jacob Berzelius é um dos fundadores da química moderna e idealizador de seus novos conceitos fundamentais, na sua época. Foi seguidor das ideias de Lavoisier e o seu interesse pela química fez com que procurasse Johan Afzelius que, mais tarde, foi seu orientador na tese de doutorado. Foi médico, professor, farmacêutico e botânico. Formou-se em 1799 e doutorou-se três anos após ambos em Medicina e, mais tarde, estudou eletroquímica. Em 1806 foi expositor de química na Academia Real de Guerra, professor de medicina e farmácia.

Berzelius foi um dos químicos mais importantes do século XIX, suas contribuições científicas foram fundamentais para o entendimento da Química e o estabelecimento de padrões para o trabalho experimental. Mesmo que muitas de suas ideias tenham sido modificadas ou provadas erradas, preparou o terreno para o desenvolvimento da química contribuindo com estudos que diziam que a química deveria ser conduzida sobre uma base estequiométrica rigorosa (Wisniak, 2000).

Dentre as descobertas deste químico, Wisniak (2000) comenta que Berzelius foi responsável pelas descobertas dos elementos cério, selênio e tório, foi o primeiro a isolar silício, cálcio, bário, estrôncio, tântalo e zircônio na forma pura, como também originou o sistema atual de nomenclatura química. Suas "Revisões Anuais" eram conhecidas como o relatório mais abrangente sobre o progresso da química e da física e entre outras conquistas, as quais incluem a introdução dos conceitos de catálise, isomerismo e alotropia.

Antes mesmo de Berzelius, os químicos Justus Von Liebig e Friedrich Wöhler já haviam relatado a existência dos primeiros isômeros: fulmanato de prata e cianato de prata. Esses cientistas verificaram que as fórmulas das duas moléculas eram idênticas (AgCNO), mas a posição dos ligantes eram diferentes, concluindo então que o arranjo espacial dos átomos era diferente (Neto, Campos \& Marcelino (2013) 
Já em 1830, o químico Berzelius, em seus experimentos para obter ácido tartárico, observou durante o processo que era possível obter duas formas diferentes da substância, com composição e fórmulas idênticas, apenas diferenciando-se pela posição dos átomos na molécula, dando nome a este fenômeno de isomeria (Fonseca, 2001).

A descoberta do fenômeno da isomeria mostrou que as propriedades das substâncias químicas não dependem unicamente de sua composição, mas também do arranjo espacial dos átomos da molécula. Sendo assim, identificaram-se as diferentes propriedades químicas e físicas de compostos formados pelos mesmos elementos e nas mesmas proporções, fato que tornou possível esclarecer na atualidade muitos problemas de âmbito científico.

\section{A Importância da Isomeria}

Segundo Atkins e Jones (2012), é comum na Química Orgânica que uma mesma fórmula molecular represente dois ou mais compostos diferentes. Esse fato é conhecido como isomeria ou isomerismo, e os diferentes compostos que tem em comum uma mesma fórmula molecular são denominados isômeros.

As diferenças entre os compostos podem ser observadas na fórmula estrutural plana, assim, são chamados de isômeros constitucionais, e, em outros casos, as diferenças entre os isômeros são percebidas ao analisarmos a estrutura espacial das moléculas com diferentes arranjos no espaço e, nesse caso, serão chamados estereoisômeros ou isômeros espaciais (Atkins \& Jones, 2012).

$\mathrm{Na}$ isomeria plana, verifica-se a diferença entre os isômeros através do estudo de suas fórmulas estruturais planas, existindo cinco casos de isomerias: de função, de cadeia, de posição, de compensação (ou metameria) e tautomeria. E na isomeria espacial, também conhecida por estereoisomeria, podendo ser de dois tipos: espacial geométrica ou óptica.

Devido ao caráter abstrato dos conteúdos sobre isomeria molecular, os professores de química orgânica enfrentam muitas dificuldades para a colocação do assunto e assim, consequentemente, os alunos compreendem muito pouco. Portanto, espera-se que os fatos históricos apresentados aqui neste trabalho ajudem a esclarer a sua importância colaborando assim por um melhor desempenho no ensino-aprendizagem.

Neto, Campos e Marcelino (2013) reforçam que os isômeros se parecem e podem até serem confundidos, como acontecido no passado, que acarretou alguns perigos em tratamentos de saúde. Na década de 1960, o medicamento com efeito tranquilizante e sonífero, a Talidomida, foi prescrito a gestantes. No entanto, pouco se sabia sobre os efeitos colaterais que essa medicação poderia causar ao feto durante a gestação. Em pouco tempo de prescrição, registrou-se um aumento de nascimentos de bebês com membros atrofiados, resultado do efeito teratogênico do isômero "S" presentes no composto da Talidomida, causando traumas irreversíveis às usuárias desta medicação e acarretando na proibição de seu uso. Na época era impossível realizar a separação de isômeros, mas atualmente a farmacologia e a química estão avançadas tecnologicamente não mais cometendo equívocos, como os do passado,

Depois do trágico episódio da Talidomida, sabe-se agora que, dependendo do isômero, podem ser esperados efeitos diferentes nos organismos vivos, daí a importância do estudo da isomeria. A diferenciação entre isômeros é constatada através da análise das suas estruturas moleculares, por apresentarem centros assimétricos capazes de produzirem enantiômeros com atividades farmacológicas diferentes, portanto, é uma análise imprescindível para a produção de medicamentos e produtos químicos seguros (Neto, Campos \& Marcelino, 2013) como acontece em fármacos como a penicilina, a adrenalina, o salbutamol entre outros (Lima, 1997). 


\section{Epistemologia de Larry Laudan e Descoberta da Isomeria por Berzelius}

Se uma teoria é metodologicamente superior a uma norma vigente da época, esse aspecto racional já pode ser suficiente para justificar sua aceitação, sem que haja necessidade de recorrer ao contexto social ou a características psicológicas dos cientistas. Por outro lado, a aceitação de uma teoria que apresenta falhas em sua fundamentação, contradizendo a norma é, muitas vezes, observada como não racional, e requer a busca de outras razões para explicá-la (Pulido \& Porto, 2015).

Fenômenos como isomeria e polimorfismo demonstraram, a sua época, que o arranjo dos átomos em uma molécula conferiria propriedades aos compostos. Os químicos não podiam mais permanecer indiferentes à maneira como os átomos elementares estavam ligados entre si nos corpos compostos e a constituição do radical deveria ser considerada (Camel, Koehler, \& Filgueiras, 2009).

Quando Berzelius, por meio de suas sínteses orgânicas, trouxe sentido para as descobertas de Liebig e Wohler, e chamou de isomeria (Esteban, 2008) foi respondida uma pergunta, portanto, foi resolvido um problema e, conforme Laudan, a Ciência progride à medida que se resolve problemas.

A partir da perspectiva de Laudan, pode-se concluir que Berzelius foi singular em suas descobertas em relação a outros pesquisadores, uma vez que conseguiu perceber as diferenças nas propriedades dos compostos considerados isômeros apesar destes compostos apresentarem fórmula molecular idêntica.

Sendo problema empírico qualquer coisa do mundo natural que nos surpreenda como estranha e que necessite de uma explicação (Laudan, 1986), pode-se dizer que Liebeg e Wohler responderam este questionamento, mas somente quando Berzelius mais tarde conceituou tal teoria ela se tornou sólida e vigente, contemplando a teoria das tradições rivais e respondendo um problema conceitual que é um problema apresentado por alguma teoria.

Laudan (1986) entende que a História da Ciência mostra que muitos dos grandes debates entre os cientistas defensores de teorias rivais ocorrem no campo conceitual, ainda que os problemas conceituais não tenham sido tema nos principais modelos epistemológicos, sendo este um dos meios importantes que a Ciência utiliza para progredir.

Trazendo estes fatos para sala de aula e levando em consideração as Orientações Curriculares Nacionais para o Ensino Médio (Brasil, 2006), que afirmam que ao estudante deve ser possibilitada a compreensão não só dos processos químicos em si como também a construção de um conhecimento científico, a Isomeria apresenta-se como um importante e complexo conteúdo a ser tratado na Química Orgânica. Sendo assim, a História da Ciência juntamente com a metodologia de Resolução de Problemas podem trazer uma melhor compreensão e proporcionar maior sentido a este conteúdo, buscando na sua origem o entendimento para a construção deste conhecimento.

\section{Considerações Finais}

Neste trabalho, apresentou-se a forma como Jacob Berzelius conduziu seus experimentos sobre isomeria e como isso representou um avanço para a Química, identificando-se em seus estudos comprometimento com a Ciência, revelando a importância de uma formação abrangente, superando a tradição dominante e provando-se que quando se busca respostas está se buscando o progresso da Ciência.

Larry Laudan, com a proposição de análise a partir da Resolução de Problemas, nos demonstra como as teorias devem ser compreendidas, na perspectiva de que a Ciência progride à medida que se resolve problemas. Desta forma, observou-se que a Resolução de Problemas a partir da descoberta de Jacob Berzelius, diante dos compostos chamados isômeros, trouxe para a atualidade benefícios de segurança tanto comerciais, científicos como sociais. 
Assim sendo, a partir do olhar de Larry Laudan, pode-se concluir que Jacob Berzelius foi singular em suas descobertas em relação a outros pesquisadores, identificando as diferenças das propriedades físicas e químicas dos compostos considerados isômeros embora estes compostos apresentem fórmulas moleculares idênticas.

A partir de aproximações e referências, pode-se encontrar uma maneira de compreensão do conteúdo isomeria em sala de aula, tornando sua maneira de ser abordada mais apropriada à situação escolar, aproximando a História da Ciência, com a Epistemologia.

Desse modo, é possível afirmar que as concepções de Larry Laudan associadas aos fatos históricos de Jacob Berzelius podem ressignificar a forma de ensinar o conteúdo de Isomeria em sala de aula, contribuindo para a evolução dos processos de ensino e de aprendizagem. A perspectiva de uma aprendizagem por Resolução de Problemas associada a conhecimentos da história das Ciências pode despertar a curiosidade dos estudantes e desenvolver outras habilidades fundamentais para o seu processo de aprendizagem, tornando-se relevante ser estudada em componentes curriculares, em programas de formação de professores, tanto na formação inicial como na continuada, e em projetos de pesquisa e de extensão, para que essa temática seja permeada por outros conteúdos relevantes no ensino de Química e contribua para uma melhor formação dos alunos.

\section{Referências}

Atkins, P. W. \& Jones, L. (2012). Princípios de Química: questionando a vida moderna e o meio ambiente. (5a ed.), Ed. Bookman.

Brasil. Ministério da Educação. (2006). Orientações curriculares Nacionais. MEC/SEF.

Camel, T. O., Koehler, C. B. G. \& Filgueiras, C. A.L. (2009). A química orgânica na consolidação dos conceitos de átomo e molécula. Química Nova, 32(2). https://doi.org/10.1590/S0100-40422009000200045.

Cervo, A. L. \& Bervian, P. A. (2002). Metodologia científica. (5a ed.), Prentice Hall.

Correa, M. E.A., Freitas, J. C.R; Freitas, J J.R. \& Filho, J. R.F. (2010). Investigação do fenômeno de isomeria: concepções prévias dos estudantes do ensino médio e evolução conceitual. In: Ensaio: Pesquisa em Educação em Ciências. Faculdade de Educação da Universidade Federal de Minas Gerais On-line version

Esteban, S. (2008). Liebig-Wöhler Controversy and the Concept of Isomerism. Journal of Chemical Education, 85(9). 1201 - 1203. http://ramsey1.chem.uic.edu/chem494/page7/files/Fulminates-Isomerism.pdf-1203.

Fonseca, M. R. M. (2001). Completamente Química: Química Orgânica.: FTD.

GIL, A. C. (2007). Como elaborar projetos de pesquisa. (4a ed.), Atlas.

Kuhn, T. (1978). The Structure of Scientific Revolutions. Chicago: The University of Chicago Press. (2a ed.), 1-222. https://www.lri.fr/ mbl/Stanford/CS477/papers/Kuhn-SSR-2ndEd.pdf.

Laudan, L. (1977). Progress and its problems. Towards a Theory of Scientific Growth. Ed. Routledge \& Kegan Paul.

Laudan, L. (1986). El progreso y sus problemas: Hacia una teoría del crecimiento científico. 1ed. Ed. Encuentro Ediciones. Madrid.

Lakatos, I. (1989). The Methodology of Scientific Research Programmes. Philosophical Papers V(1). Cambridge University Press. 1-256. http://strangebeautiful.com/other-texts/lakatos-meth-sci-research-phil-papers-1.pdf.

Leite, L. F. C. C., Silva, A. L., \& Lins, R. (2019). Uma Webquest para facilitar o ensino de isomeria óptica. In: M. B. Justus (Org.) Formação de Professores e a Condição do Trabalho Docente 2(22), 223-229. Ed. Atena.

Lima, V. L.E. (1997). Os Fármacos e a Quiralidade: uma breve abordagem. Química Nova, 20(6), 657-663. https://www.scielo.br/j/qn/a/kLchnKvkHLBthT dyBRRTvbx/?format=pdf\&lang=pt.

Lüdke, M. \& André, M. E.D.A. (1986). Pesquisa em Educação: Abordagens Qualitativas. Ed. Pedagógica e Universitária - EPU.

Marcelino, C. A. C. Barbosa, R. M. N. Campos, A. F., Leão, M. B.C., Cunha, H. S. \& Pavão, A. C. (2010). Perfumes e essências: a utilização de um vídeo na abordagem das funções orgânicas. Química Nova na Escola, 19(1) 15-18. http://qnesc.sbq.org.br/online/qnesc19/a05.pdf.

Medeiros, D. R. Silva, E. R.A. Goi, M. E. J. Gonçalves, P. (2020). Uma Análise a partir da Epistemologia de Larry Laudan dos modelos atômicos. Cadernos CIMEAC. 10(2). 10.18554/cimeac.v10i2.3773.

Moreira, M. A. \& Massoni, N. T. (2011). Epistemologias do século XX. Editora Pedagógica Universitária Ltda. 
Neto, J. E. S. Campos, A. F. C \& Marcelino, C. A. C. (2013). Abordando a Isomeria em Compostos Orgânicos e Inorgânicos: Uma atividade fundamentada no uso de situações Problemas na Formação Inicial de Professores de Química. Investigações em Ensino de Ciências. 18(2). 327-346. file://C:/Users/user/Downloads/133-264-1-SM\%20(1).pdf.

Ostermann, F. Cavalcanti, C. J. H., Ricci, T. F. \& Prado, S. D. (2008). Tradição de pesquisa quântica: uma interpretação na perspectiva da epistemologia de Larry Laudan. Revista Electrónica de Enseñanza de las Ciencias, 7(2). 366-386. http://reec.uvigo.es/volumenes/volumen7/ART6_Vol7_N2.pdf.

Pulido, M. D. \& Porto, P A. (2016). O dualismo eletroquímico de Berzelius: sua caracterização e presença em livros didáticos de química. Ensino de Ciências (Física, Química e Biologia), Universidade de São Paulo. 10.11606/D.81.2016.tde-25112016-151011.

Santos, F. M. T. \& Goi, M. E. J. (2012). Resolução de Problemas no Ensino de Química fundamentos epistemológicos para o emprego da metodologia na Educação Básica. In Anais do XVI Encontro Nacional de Ensino de Química / X Encontro de Educação Química da Bahia. Salvador: Editora da Universidade Federal da Bahia, 1-11. https://www.ufrgs.br/forprof-ciencias/editais/texto3.pdf.

Santana, W. P. \& Lemos, G. C. (2018). Metodologia Científica: a pesquisa qualitativa nas visões de Lüdke e André. Revista Eletrônica Científica Ensino Interdisciplinar Mossoró, 4(12). http://natal.uern.br/periodicos/index.php/RECEI/article/view/1710.

Vieira, U.S. Barbosa, A. S. Muribeca, A. J. B. \& Gomes, P. W. P. (2019). O Pecíolo do Burití como instrumento no ensino de Isomeria Constitucional e Estereoisomeria. Revista Ciências \& Ideias 10(1): 01 10.22407/2019.v10i1.873.

Vilela, M. V. F. Rocha, E. F. R. Silva, V.C. Castro, E.B. Araújo, C S. O. (2021). Reflexões Históricas e Epistemológicas sobre a Trajetória da Ciência e suas Implicações para o Ensino de Ciências: Contribuições do Estudo de Temas CTS à luz da HFC em prol da superação de imagens distorcidas do trabalho científico. Research, Society and Development. 10(9). https://doi.org/10.33448/rsd-v10i9.18422.

Wisniak, J. (2000). Jöns Jacob Berzelius Um guia para o químico perplexo. The Chemical Educator 5(6), p 343-350. 10.1007 / s00897000430a. 